\title{
No evidence for an involvement of copy number variation in $A B C A 13$ in schizophrenia, bipolar disorder, or major depressive disorder
}

\author{
Franziska Degenhardt ${ }^{\mathrm{a}, \mathrm{b}}$, Lutz Priebe ${ }^{\mathrm{a}, \mathrm{b}}$, Jana Strohmaier ${ }^{\mathrm{f}}$, Stefan Herms ${ }^{\mathrm{a}, \mathrm{b}}$, \\ Per Hoffmann ${ }^{\mathrm{a}, \mathrm{b}}$, Manuel Mattheisen ${ }^{\mathrm{b}, \mathrm{c}, \mathrm{l}}$, Rainald Mössner ${ }^{\mathrm{d}}$, Igor Nenadic ${ }^{\mathrm{g}}$, \\ Heinrich Sauer ${ }^{g}$, Dan Rujescu ${ }^{h, i}$, Wolfgang Maier ${ }^{d}$, Thomas G. Schulze ${ }^{\mathrm{f}, \mathrm{j}}$, \\ Marcella Rietschel ${ }^{f}$, Markus M. Nöthen ${ }^{\mathrm{a}, \mathrm{b}, \mathrm{e}}$ and Sven Cichon ${ }^{\mathrm{a}, \mathrm{b}, \mathrm{k}}$
}

\section{Psychiatric Genetics 2013, 23:45-46}

anstitute of Human Genetics, b ${ }^{\text {a }}$ Department of Genomics, Life and Brain Center, Institute for Genomic Mathematics, dDepartment of Psychiatry, University of Bonn, ${ }^{e}$ German Center for Neurodegenerative Diseases (DZNE), Bonn, fDepartment of Genetic Epidemiology in Psychiatry, Central Institute of Mental Health, University of Heidelberg, Mannheim, ${ }^{9}$ Department of Psychiatry and Psychotherapy, Jena University Hospital, Jena, ${ }^{\mathrm{h}}$ Molecular and Clinical Neurobiology, Department of Psychiatry, Ludwig-Maximilians University, Munich, 'Department of Psychiatry, University of Halle-Wittenberg, Halle, 'Section of Psychiatric Genetics, Department of Psychiatry and Psychotherapy, University Medical Center, Georg-August University, Göttingen, Germany, 'Institute of
Neuroscience and Medicine (INM-1), Structural and Functional Organisation of the Brain, Genomic Imaging, Research Centre Juelich, Juelich, Germany and 'Channing Division of Network Medicine, Brigham and Women's Hospital and Harvard Medical School, Boston, Massachusetts, USA

Correspondence to Sven Cichon, PhD, Department of Genomics, Life and Brain Center, Sigmund-Freud-Straße 25, 53127 Bonn, Germany Tel: + 492286885 405; fax: +492286885 401; e-mail: sven.cichon@uni-bonn.de

Received 21 June 2012 Accepted 2 July 2012

All individuals were genotyped on HumanHap550v3, Human610-Quadv1, and Human660W-Quad arrays (Illumina, San Diego, California, USA). Detailed information on CNV detection and CNV quality control is provided elsewhere (Degenhardt $e t$ al., 2012). Only those markers common to all three chips were analyzed. Participants were excluded if their SD from the $\log R$ ratio calculated over all SNPs exceeded 0.30.

The three data sets were analyzed for the presence of both microdeletions and microduplications including the $A B C A 13$ gene plus $10 \mathrm{~kb}$ upstream and downstream of the Refseq gene boundary (chr7: 48208389-48657637; hg18). All CNVs were required to span a minimum of 10 consecutive SNPs and have a log Bayes Factor (QuantiSNP) or confidence value (PennCNV) of at least 10 .

No CNV including $A B C A 13$ was identified in our three patient samples, nor in the controls. Hence, we did not find any evidence for $\mathrm{CNV}$ s in $A B C A 13$ as a risk factor for the development of SCZ, BPD, or MDD. It cannot be excluded, however, that extremely rare $\mathrm{CNV}$ s might play a role in disease development, which would only be detectable in considerably larger samples. It is therefore important to gather data from several large studies before final conclusions regarding the involvement of CNVs in $A B C A 13$ in SCZ, BPD, or MDD can be drawn.

\section{Acknowledgements}

The authors thank all the patients for participating in this study. They are grateful to Professor H.E. Wichmann for supplying the SNP-chip data from the KORA control cohort and to Professor S. Schreiber for providing access to the SNP-chip data from the PopGen control cohort. 
They acknowledge the support of Christine Esslinger, Claudia Schütz, Nina Seiferth, Sebastian Mohnke, and Leila Haddad in the recruitment of the MooDS controls. They thank all the probands from the Heinz Nixdorf Recall (HNR) study, and the MooDS Imaging controls.

This study was supported by the German Federal Ministry of Education and Research (BMBF), within the context of: the National Genome Research Network 2 (NGFN-2); the National Genome Research Network plus (NGFNplus); and the Integrated Genome Research Network (IG) MooDS (Grant 01GS08144 to S.C. and M.M.N., Grant 01GS08147 to M.R.). In addition, this work was supported by the European Union grant number HEALTH-F4-2009242257 (Project ADAMS). J.S. was supported by the German Research Foundation (GRK 793). M.M.N. also received support from the Alfried Krupp von Bohlen und Halbach-Stiftung. The HNR cohort was established with the support of the Heinz Nixdorf Foundation. I.N. was supported by a Junior Scientist grant of the Interdiziplinäres Zentrum für Klinische Forschung Jena (IZKF).

\section{Conflicts of interest}

There are no conflicts of interest.

\section{References}

Degenhardt F, Priebe L, Herms S, Mattheisen M, Mühleisen TW, Meier S, et al. (2012). Association between copy number variants in $16 \mathrm{p} 11.2$ and major depressive disorder in a German case-control sample. Am J Med Genet $B$ Neuropsychiatr Genet 159B:263-273.

Knight HM, Pickard BS, Maclean A, Malloy MP, Soares DC, Mcrae AF, et al. (2009). A cytogenetic abnormality and rare coding variants identify ABCA13 as a candidate gene in schizophrenia, bipolar disorder, and depression. Am J Hum Genet 85:833-846.

Pickard BS, Van Den Bossche MJ, Malloy MP, Johnstone M, Lenaerts AS, Nordin A, et al. (2012). Multiplex amplicon quantification screening the ABCA13 gene for copy number variation in schizophrenia and bipolar disorder. Psychiatr Genet [Epub ahead of print].

Priebe L, Degenhardt FA, Herms S, Haenisch B, Mattheisen M, Nieratschker V, et al. (2012). Genome-wide survey implicates the influence of copy number variants (CNVs) in the development of early-onset bipolar disorder. Mol Psychiatry 17:421-432.

Rietschel M, Mattheisen M, Degenhardt F, Kahn RS, Linszen DH, Os JV, et al. GROUP Investigators Genetic Risk and Outcome in Psychosis (GROUP Investigators) (2011). Association between genetic variation in a region on chromosome 11 and schizophrenia in large samples from Europe. Mol Psychiatry [Epub ahead of print]. 